\title{
Estigmatização e Prática de Profissionais da APS Referentes ao Consumo de Álcool
}

\author{
Stigmatization and APS's Professional Practice \\ in Relation to the use of Alcool
}

Estigmatización y Práctica de Profesionales de la APS Referentes al Consumo de Alcohol

Marina Castro de Oliveira \& Telmo Mota Ronzani

Universidade Federal de Juiz de Fora
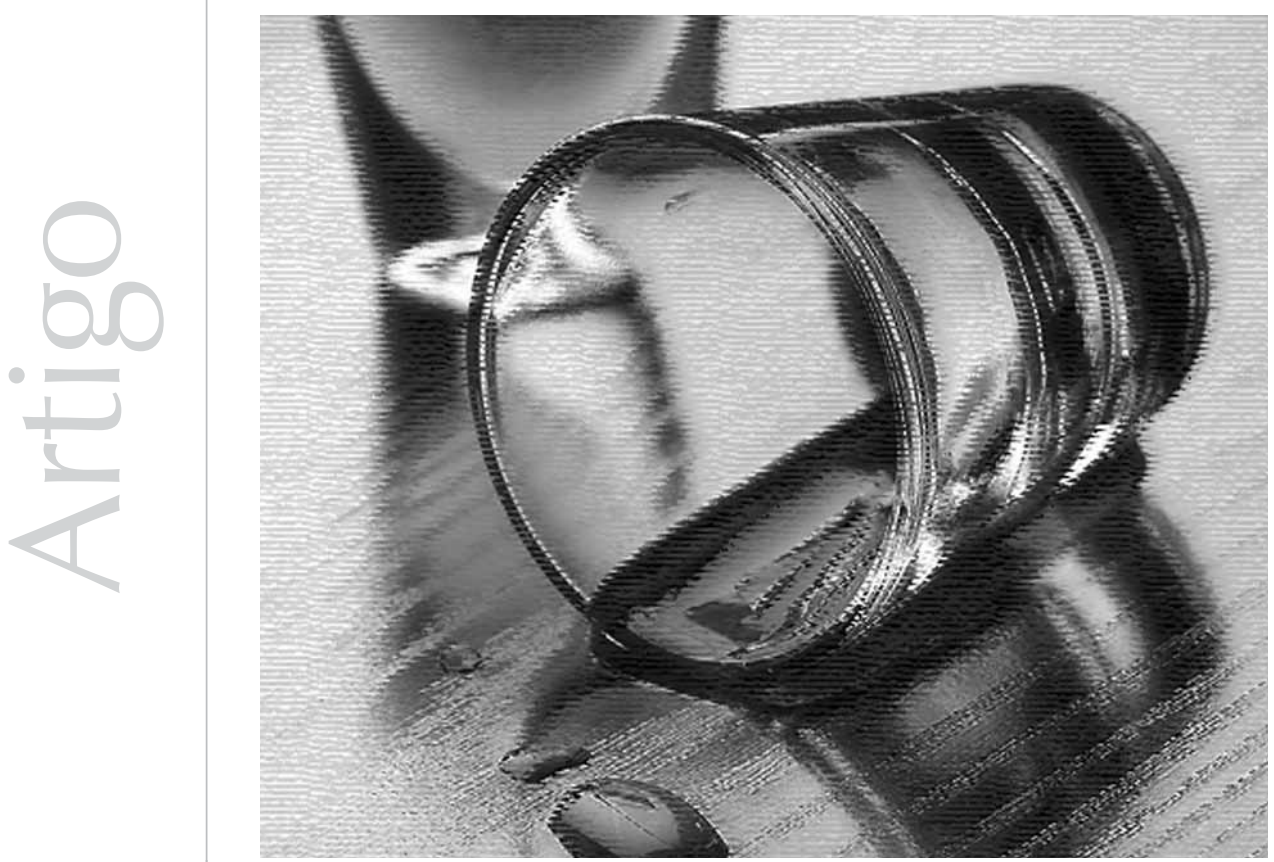
Resumo: Uma das condições de saúde mais estigmatizada no mundo é a dependência de álcool, sendo essa estigmatização apontada pela literatura como uma considerável barreira à efetiva atenção em saúde a esses pacientes. O objetivo do presente artigo foi avaliar a associação entre a estigmatização do alcoolismo pelos profissionais da Atenção Primária à Saúde e as suas práticas em relação ao consumo de álcool. Os instrumentos utilizados foram: Questionário de Práticas de Prevenção ao Uso de Álcool, a fim de verificar a frequência com que tais práticas ocorrem, e o Questionário de Modelo de Percepção do Uso de Álcool, a fim de avaliar a estigmatização relativa ao alcoolismo. Participaram do estudo 170 profissionais de três cidades de pequeno porte da Zona da Mata mineira. Verificou-se que o alcoolismo é uma condição estigmatizada, entretanto, observou-se que os profissionais que estigmatizam ou que não moralizam o alcoolismo realizam práticas direcionadas ao consumo de álcool com a mesma frequência. Dessa forma, sugere-se que estudos futuros avaliem as práticas dos profissionais de uma forma mais ampla, a fim de identificar como a estigmatização se associa a tais atividades.

Palavras-chave: Alcoolismo. Abuso de álcool. Estigma. Profissionais de saúde. Atenção Primária à Saúde.

\begin{abstract}
Alcoholism is one of the most stigmatized health conditions of the world, and this stigma is pointed as a significant barrier to provide service to patients. The aim of this paper was to evaluate the association between the stigma of alcoholism by primary health care professionals and their practices related to alcohol consumption. The study included 170 professionals from three small towns in Zona da Mata mineira, Brazil. Two instruments were used: a Preventive Practice for Alcohol Use Questionnaire, in order to determine the frequency in which these practices occur and Perception Model for Alcohol Use Questionnaire, with the purpose of evaluating the moralization in relation to alcoholism. The fact that alcoholism is a stigmatized condition was observed, however, professionals who moralize and who do not moralize alcoholism conduct practices of alcohol consumption with the same frequency. Thus, it is suggested that future studies should evaluate the practices using a broader method to identify how stigma is associated with such activities.
\end{abstract}

Keywords: Alcoholism. Alcohol abuse. Stigma. Health care professionals. Primary Health Care.

Resumen: Una de las condiciones de salud más estigmatizada en el mundo es la dependencia de alcohol, siendo esa estigmatización apuntada por la literatura como una considerable barrera a la efectiva atención en salud a esos pacientes. El objetivo del presente artículo fue evaluar la asociación entre la estigmatización del alcoholismo por los profesionales de la Atención Primaria a la Salud y sus prácticas en relación al consumo de alcohol. Los instrumentos utilizados fueron: Cuestionario de Prácticas de Prevención al Uso de Alcohol, a fin de verificar la frecuencia con que tales prácticas ocurren, y el Cuestionario de Modelo de Percepción del Uso de Alcohol, a fin de evaluar la estigmatización relativa al alcoholismo. Participaron del estudio 170 profesionales de tres ciudades de pequeño porte de la Zona da Mata minera. Fue verificado que el alcoholismo es una condición estigmatizada, no obstante, se observó que los profesionales que estigmatizan o que no moralizan el alcoholismo realizan prácticas direccionadas al consumo de alcohol con la misma frecuencia. De esa forma, se sugiere que estudios futuros evalúen las prácticas de los profesionales de una forma más amplia, a fin de identificar cómo la estigmatización se asocia a tales actividades.

Palabras clave: Alcoholimo. Abuso del alcohol. Estigma. Profesionales de salud. Atención Primaria a la Salud.

O consumo de álcool é prevalente em todo o mundo, sendo considerado um relevante problema de saúde pública. Dados indicam que os transtornos relacionados ao consumo de álcool representam uma das dez principais condições de saúde que contribuem para explicar os anos de vida perdidos por mortes prematuras entre a população adulta (World Health Organization, 2003).
A dependência ao álcool está em quinto lugar entre os principais problemas de saúde que atingem a população de 15 a 44 anos em todo o mundo em termos de Anos de Vida Ajustados por Incapacidade (AVAI), um indicador de saúde desenvolvido pela Organização Mundial de Saúde (OMS) para avaliar a qualidade de vida da população (World Health Organization, 
1999). No Brasil, de acordo com o Segundo Levantamento Domiciliar sobre o uso de Drogas Psicotrópicas, o alcoolismo atinge $12,3 \%$ da população (Carlini, Galduróz, Noto, \& Nappo, 2005).

Entretanto, os malefícios do uso do álcool não se restringem aos ocasionados pela dependência. A maior parte dos danos relacionados ao consumo dessa substância pode ser atribuída a um grupo de usuários mais amplo, constituído pelo consumo de risco e pelo consumo nocivo (Humeniuk \& Poznyak, 2004). O consumo de risco é um padrão que aumenta as chances de danos futuros para os usuários e para os que o cercam, porém ainda não pressupõe danos ao indivíduo. Já o consumo nocivo se refere a um padrão que já acarreta danos físicos e mentais à saúde do indivíduo, além de consequências em nível social (Babor, Higgins-Biddle, Saunders, \& Monteiro, 2001).

O consumo nocivo de álcool é responsável por $3,7 \%$ de todas as mortes e está entre as principais causas evitáveis de morbimortalidade no mundo, tendo relação direta com problemas físicos, sociais e psicológicos, tais como câncer, condições neuropsiquiátricas, condições cardiológicas, gastrintestinais, diferentes formas de violência como a violência doméstica e o abuso infantil, além de acidentes de trânsito e suicídios, entre outros (World Health Organization, 2007).

No Brasil, o consumo de álcool é responsável por $10 \%$ da morbimortalidade do País. Estudos revelam que, além do enorme impacto na saúde, o consumo de álcool também está relacionado diretamente a diversos problemas sociais brasileiros, tais como vandalismo, desordem pública, problemas familiares, como conflitos conjugais e divórcio, abuso de menores, problemas interpessoais, problemas financeiros, problemas ocupacionais, dificuldades educacionais, acidentes de trânsito e custos sociais (Senad, 2007; Hoffmann, Carbonell, \& Montoro, 2006; Laranjeira \& Meloni, 2004).

Apesar da magnitude dos problemas oriundos do consumo de álcool, poucas ações em saúde são realizadas visando aos alcoolistas ou consumidores abusivos e de risco. Existem diversos fatores que contribuem para a falta de diagnóstico, tratamento ou encaminhamento das pessoas que apresentam complicações decorrentes do consumo de álcool, dentre elas, a falta de conhecimento sobre a variedade de apresentações sintomáticas geradas pelo uso abusivo e pela dependência do álcool, e também uma visão negativa, estigmatizada, em relação ao paciente e às suas perspectivas evolutivas frente ao problema (Brasil, 2004).

\section{Implicações da estigmatização do consumo de álcool pelos profissionais de saúde}

Muitas condições de saúde, dentre elas, a saúde mental, são estigmatizadas pela população em geral, incluindo os profissionais de saúde (Berger, Wagner, \& Baker, 2005), sendo o consumo de álcool e outras drogas uma das condições mais estigmatizadas (Palm, 2006; Corrigan, Lurie, Goldman, Slopen, Medasani, \& Phelan, 2005).

O termo estigma pode ser definido como marca física ou social de conotação negativa ou que leva o portador dessa marca a ser marginalizado ou excluído de algumas situações sociais. A estigmatização ocorre quando se atribuem rótulos e estereótipos negativos a determinados comportamentos (Ronzani \& Andrade, 2006).

No campo da saúde, o uso de estereótipos, ou seja, a atribuição de características pessoais ou sociais a determinadas pessoas ou grupos antes mesmo de ser realizada uma 
..muitas vezes, em situações de saúde, a condição de estigmatização pode ser muito mais danosa do que a doença em si (Ronzani et al. 2009), e pode contribuir significativamente para a perda da qualidade de vida desses pacientes (Peluso \& Blay, 2008). avaliação cuidadosa, podem ser observados na classificação do comportamento das pessoas de acordo com o problema de saúde ou com a marca que lhes é conferida. Essa tendência é prejudicial tanto ao profissional, que deixa de perceber ou conhecer o problema mais a fundo, quanto ao paciente, que deixa de receber uma intervenção adequada. Tal situação influencia direta ou indiretamente a condição de saúde da pessoa estigmatizada, o que pode provocar, entre outras consequências, o agravamento da situação (Ronzani \& Andrade, 2006; Berger et al., 2005).

Um dos processos cognitivos envolvidos na estigmatização é a atribuição moral, que seria uma forma de atribuição de causalidade sobre um comportamento observado (Ronzani, Higgins-Biddle, \& Furtado, 2009). De acordo com o modelo de percepção de Brickman, apresentado por Palm (2006), a atribuição moral ocorre quando um indivíduo é visto como responsável pelo aparecimento e pela solução de seu próprio problema.

No processo de formação do estigma social, as características negativas atribuídas a determinada condição são reconhecidas não somente pelo grupo externo mas também internalizadas pelas pessoas portadoras desse estigma, o que influencia diretamente as crenças e os sentimentos das pessoas em relação às características atribuídas como negativas (Ronzani \& Andrade, 2006). Nesse sentido, muitas vezes, em situações de saúde, a condição de estigmatização pode ser muito mais danosa do que a doença em si (Ronzani et al. 2009), e pode contribuir significativamente para a perda da qualidade de vida desses pacientes (Peluso \& Blay, 2008).

Estudos apontam que as pessoas que possuem uma doença mental são vistas como mais responsáveis por sua condição do que as pessoas que sofrem de outras doenças como câncer e problemas cardíacos (Corrigan et al. 2005). Estudos também indicam que a maior parte dos profissionais que lidam com o tratamento de dependentes de álcool e outras drogas acredita que seus pacientes são parcialmente responsáveis pelo aparecimento do problema e totalmente responsáveis pela solução do mesmo, ou seja, apresentam uma percepção moral em relação ao usuário (Palm, 2006). A predominância da percepção moral também foi verificada entre os profissionais de saúde da Atenção Primária à Saúde (APS) (Silveira, Martins, \& Ronzani, 2009; Ronzani et al., 2009).

Diante do impacto que o processo de estigmatização acarreta sobre os serviços de saúde, o comitê especialista em problemas relacionados ao consumo de álcool sugere que a Organização Mundial de Saúde (OMS) estimule mais estudos nessa área, com particular atenção sobre a forma como a redução da estigmatização pode ser levada em conta em políticas sobre o álcool (World Health Organization, 2007).

A literatura aponta três formas principais de redução de estigma, daí a importância da formação continuada e de capacitações direcionadas aos profissionais de saúde.

\section{Avaliação da estigmatização do consumo de álcool na APS}

A APS no Brasil é organizada pelo Sistema Único de Saúde - SUS, que adota como estratégia prioritária o Programa de Saúde da Família - PSF, que abrange ações de promoção, prevenção, reabilitação e tratamento (Andrade, Barreto, \& Bezerra, 2006). A implantação do PSF em 1994 é um marco na incorporação da estratégia de atenção primária na política de saúde brasileira; trata-se de uma iniciativa recente que revela a carência de estudos destinados a avaliar a efetividade de suas ações (Escorel, 
Giovanella, Mendonça, \& Castro, 2007), incluindo aquelas voltadas para o consumo de álcool.

A APS é o nível de atenção à saúde que atinge a maior parte da população, o que ressalta sua condição estratégica dentro do sistema de saúde brasileiro. Nos Municípios de pequeno porte, o impacto da APS pode ser ainda maior, se considerarmos que, nesses Municípios, ela é a principal - senão única - forma de oferta de serviços públicos em saúde (Ronzani \& Andrade, 2006).

O Ministério da Saúde reconhece que os profissionais da APS são essenciais para o desenvolvimento de ações de prevenção primária ao uso prejudicial do álcool, para o diagnóstico precoce, desenvolvimento de ações de redução de danos, tratamento de quadros não complicados e referenciamento dos quadros moderados e graves para a rede de assistência (Brasil, 2004).

Dessa forma, a política do Ministério da Saúde para a Atenção Integral a Usuários de Álcool e outras Drogas (Brasil, 2004), em consonância com a literatura científica, ressalta a importância de se ampliar as atividades do Programa Permanente de Capacitação de Recursos Humanos para os Serviços de Atenção aos Usuários de Drogas na Rede do SUS, capacitando não apenas profissionais especializados como também os que trabalham em Centro de Atenção a Usuários de Substâncias Psicoativas - CAPS ad e os da APS (PSF e os do Programa de Agentes Comunitários de Saúde -PACS).

Diante disso, o presente artigo tem como objetivo avaliar a associação entre a estigmatização do alcoolismo e as características das práticas em saúde referentes ao consumo de álcool entre profissionais da APS de Municípios de pequeno porte da Zona da Mata de Minas Gerais. Baseado na literatura (Ronzani et al., 2009; Peluso \& Blay, 2008; Palm, 2006; Corrigan et al., 2005), as principais hipóteses do estudo são que a dependência de álcool é uma condição de saúde estigmatizada (moralizada) pelos profissionais, e que essa estigmatização se associa às práticas em saúde referentes ao consumo de álcool.

\section{Metodologia}

\section{Participantes}

Participaram da pesquisa 170 profissionais da atenção primária que trabalham em programas de saúde da família e na assistência social de três Municípios de pequeno porte da Zona da Mata de Minas Gerais com menos de 40.000 habitantes.

O Município A possui uma população de 16.166 habitantes (IBGE, 2010) e quatro equipes de ESF, totalizando $x$ profissionais. $\mathrm{O}$ Município $B$ possui uma população de 9.750 habitantes (IBGE, 2010) e conta com quatro equipes de ESF que englobam $x$ profissionais. O Município $C$ possui uma população de 25.062 (IBGE, 2010) e possui seis equipes de ESF, que totalizam $x$ profissionais. A descrição da amostra total para as variáveis gênero, idade, categoria profissional, tempo de profissão e escolaridade é apresentada na Tabela 01. Destaca-se que houve uma diferença entre os gêneros e que a maior parte dos profissionais é ACS.

Tais profissionais foram convidados a participar de uma capacitação sobre práticas de prevenção ao consumo de álcool e outras drogas, e, antes do início, responderam aos questionários utilizados no presente estudo, no próprio local de trabalho. A participação foi condicionada à aceitação e à assinatura do Termo de Consentimento Livre e Esclarecido (TCLE). O projeto recebeu aprovação do Comitê de Ética em Pesquisa da Universidade Federal de Juiz de Fora, Processo no 0419 / 06. 


\section{Delineamento de pesquisa}

O presente estudo foi do tipo exploratório, não controlado, de definição intencional da amostra. Este estudo é parte de uma pesquisa mais ampla intitulada Avaliação de Processo sobre Prática de Prevenção ao Uso de Drogas e Violência Doméstica em Serviços de Atenção Primária à Saúde, da Universidade Federal de Juiz de Fora. Para melhor operacionalização das hipóteses de estudo, optou-se por avaliar dois aspectos principais: o modelo de percepção do alcoolismo e as práticas em saúde referentes ao consumo de álcool.

\section{Instrumentos}

Foram utilizados questionários estruturados administrados em forma de autoaplicação. Os instrumentos são apresentados a seguir:

\section{Questionário de Práticas de Prevenção} ao Uso de Álcool - esse questionário foi utilizado visando a avaliar a prática em saúde dos profissionais referente ao consumo de álcool (Ronzani, 2005). O questionário é composto pelas seguintes questões: 1. Qual a porcentagem dos pacientes adultos que você atende que você estima terem problemas relacionados ao uso de álcool? 2. Como parte de sua consulta a um paciente adulto, com que frequência você pergunta a ele(a) especificamente sobre seu consumo e a história dos problemas relacionados ao uso de álcool? 3. Como parte de sua consulta a um paciente adulto, com que frequência você aconselha os pacientes com padrões de consumo de riscos para problemas relacionados ao uso de álcool a diminuir ou a parar com esse consumo? 4. Nos últimos 12 meses, dos pacientes adultos que você atendeu, aproximadamente quantos você tratou dos problemas relacionados ao consumo pesado de álcool? 5. Em relação às atividades de prevenção aos problemas relacionados ao uso de álcool, em qual das proposições abaixo você melhor se encaixa? 6. Você desenvolve atividade(s) ou abordagens preventivas em relação ao consumo de álcool e outras drogas? 7. Com que frequência você desenvolve palestras ou orientações preventivas para o consumo de álcool ou outras drogas? As opções de respostas variam de acordo com a questão, apresentando de 2 a 7 opções de respostas, com exceção da primeira questão, cuja resposta é dada em porcentagem (Ronzani, 2005).

\section{Questionário sobre Modelo de Percepção} do Uso de Álcool: para mensurar o estigma social, foi avaliado um de seus aspectos, a atribuição moral do uso de álcool. Essa avaliação consiste em uma classificação simples e objetiva sobre como o profissional percebe o problema da dependência do álcool (Ronzani et al., 2009; Palm, 2006).

O questionário é composto por uma pergunta acerca da responsabilidade dos pacientes com relação ao aparecimento do alcoolismo e outra sobre a responsabilidade que esse paciente teria para solucionar esse problema. A possibilidade de resposta de concordância em uma escala de 1 a 5 , em que 1 corresponde a nada responsável e 5, a totalmente responsável, foi oferecida para as perguntas: O quanto você acha que o paciente é responsável pelo aparecimento/ evolução dos seus problemas de saúde? e O quanto você acha que o paciente é responsável pela solução dos seus problemas de saúde? De acordo com o modelo teórico de Brickman, utilizado por Palm (2006), pode haver quatro modelos de percepção sobre o uso de álcool de acordo com a responsabilização pelo desenvolvimento/ aparecimento e pela solução do alcoolismo. Os quatro modelos estão apresentados na Tabela 1 e são compreendidos da seguinte maneira:

a. Modelo moral: o próprio indivíduo é responsável pelo aparecimento e pela solução 
de determinado problema de saúde. O alcoolismo é visto como mau hábito, sendo sua causa atribuída a uma fraqueza moral, e sua não solução, à falta de força de vontade.

b. Modelo médico: o indivíduo é minimamente responsável pela causa e solução do problema, porque o alcoolismo tem uma etiologia biológica. O alcoolismo é visto como um problema irreversível e sem cura. O tratamento para o problema seria possível por meio do acompanhamento de um profissional ou programa que o auxiliasse na busca da abstinência.

c. Modelo compensatório: o indivíduo é minimamente responsável pela causa ou surgimento do problema, entretanto, é responsável por resolver seu problema desenvolvendo novas habilidades de enfrentamento ou adquirindo melhor sistema de suporte social.

d. Modelo iluminado: o indivíduo é responsável por causar seu problema, porque ele foi levado pelo impulso de beber e perder o controle. Entretanto, o alcoolista tem um controle mínimo pela resolução de seu problema. Sendo assim, o tratamento necessita que o alcoolista admita a fraqueza perante a bebida e submeta o seu controle a uma força maior que ilumine seus passos. Essa força poderia ser a comunidade ou outros alcoolistas que estão se recuperando.

Tabela 1. Modelo Brickman baseado na atribuição de responsabilidade do indivíduo pelo aparecimento e solução do problema

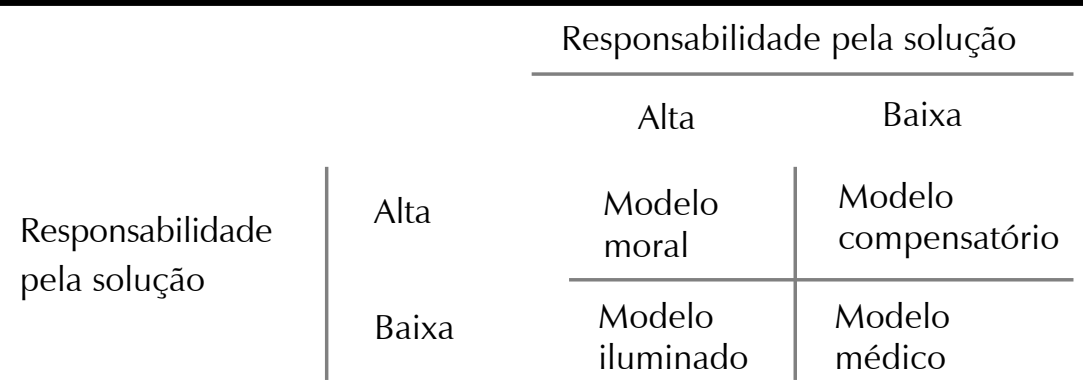

É válido ressaltar a dificuldade e o cuidado necessário para a mensuração de atitudes e o preconceito implícito, devido à tendência de as pessoas darem respostas socialmente aceitáveis. Além do modelo utilizado, uma segunda opção de mensuração construída na tentativa de driblar essa tendência são as vinhetas, instrumentos úteis para avaliar, por exemplo, as reações emocionais que os dependentes de álcool despertam, o reconhecimento da dependência como uma doença mental, as crenças acerca das causas de tal condição, a percepção de periculosidade dos dependentes e o quão desejado é o distanciamento social dessas pessoas (Silveira, 2010).

\section{Análise dos dados}

Os dados foram digitados no software estatístico SPSS versão 15.0, com a finalidade de corrigir eventuais erros de digitação e garantir a qualidade dos dados. Por meio desse mesmo software, os dados foram organizados e analisados. Inicialmente, utilizaram-se técnicas de estatística descritiva para apresentar as frequências (n), as porcentagens (\%), a média (M), a mediana, o desvio padrão (DP) e os valores mínimos (Mín.) e máximos (Máx.). A partir de então, verificou-se, pelo Teste Estatístico de Kolmogorov-Smirnov, a hipótese nula de não existência de diferença estatística 
entre a distribuição amostral das variáveis numéricas utilizadas neste estudo e uma distribuição normal. A hipótese nula foi rejeitada para todas as variáveis numéricas, portanto, optou-se por prosseguir as análises inferenciais por meio de estatísticas não paramétricas, indicando a mediana como medida de tendência central. O Teste de Mann-Whitney foi utilizado para testar a hipótese nula de não existência de diferença estatisticamente significativa entre a média dos postos das variáveis numéricas de duas amostras independentes; quando essa comparação era feita entre mais de duas amostras independentes, utilizou-se o Teste de Kurskall-Wallis. Para a análise de existência de relacionamento entre duas variáveis numéricas, utilizou-se a correlação bivariada de Spearman para analisar o coeficiente de correlação entre as variáveis ( $\square$, rho de Sperman) e testar a hipótese nula de não existência de relacionamento estatisticamente significativo entre as variáveis analisadas.

O Teste do Qui-quadrado de Pearson foi utilizado para testar a hipótese nula de não existência de diferença estatística entre a proporção da distribuição de grupos definidos a partir de variáveis nominais relacionadas. O Teste Exato de Fisher foi calculado para obter valores de significância corrigidos quando violadas as propriedades de número mínimo de observações por célula no Teste do Qui-quadrado. Adotou-se, para todas as análises inferenciais, o intervalo de confiança de $95 \%(p<0,05)$.

\section{Resultados}

A Tabela 2 apresenta a distribuição das frequências para cada modelo de percepção para a condição de alcoolista de acordo com o modelo Brickman. Houve uma diferença significativa na distribuição de frequência entre os modelos ( $p<0,001$ no Teste de Qui-quadrado). Observouse que o modelo moral apresentou a maior frequência $(78,7 \%)$, seguido pelo modelo médico $(12,8 \%)$, pelo compensatório $(5,3 \%)$ e, por último, pelo iluminado $(3,2 \%)$.

Tabela 2. Distribuição de frequência e porcentagem sobre a percepção do alcoolismo por parte de profissionais da Atenção Primária de acordo com o modelo Brickman

\begin{tabular}{lcc}
\hline Modelo & Frequência & Porcentagem \\
Médico & 12 & $12,8 \%$ \\
Compensatório & 05 & $5,3 \%$ \\
lluminado & 03 & $3,2 \%$ \\
Moral & 74 & $78,7 \%$ \\
Total & 94 & $100 \%$ \\
\hline
\end{tabular}


A tabela 3 apresenta as características das práticas realizadas pelos profissionais da APS em relação ao consumo de álcool de acordo com o questionário Caracterização de Práticas em Saúde.

Verificou-se uma mediana 20 para a porcentagem estimada de 3 pacientes atendidos com problemas relacionados ao consumo de álcool; observou-se que $64,4 \%$ dos profissionais de APS perguntam, como parte da consulta de um paciente adulto sobre seu consumo de álcool e a história dos problemas relacionados ao uso, a maioria das vezes ou algumas vezes; que $65,4 \%$ dos profissionais aconselha todas as vezes ou a maioria das vezes os pacientes com padrões de consumo de risco para problemas relacionados ao consumo de álcool a diminuir ou a parar o consumo; $68,4 \%$ dos profissionais relata ter atendido de (0-5) pacientes com consumo pesado nos últimos 12 meses; $37,7 \%$ dos profissionais "está pensando em fazer, mas ainda não começou" atividades de prevenção aos problemas relacionados ao uso de álcool; 60,2\% dos profissionais não desenvolve atividades preventivas em relação ao consumo de álcool e outras drogas, e 62,2\% dos profissionais nunca desenvolveu palestras ou orientações preventivas para o consumo de álcool e outras drogas.

Tabela 3. Práticas de saúde dos profissionais da Atenção Primária

\begin{tabular}{|c|c|c|}
\hline Questão & Resposta & Freqüência \\
\hline 1 & $\begin{array}{l}\text { Mediana da porcentagem estimada de } \\
\text { pacientes atendidos com problemas } \\
\text { relacionados ao uso de álcool }\end{array}$ & 20 \\
\hline 2 & $\begin{array}{l}\text { Pergunta sobre o consumo nas } \\
\text { consultas a maioria das vezes ou } \\
\text { algumas vezes }\end{array}$ & $64,4 \%$ \\
\hline 3 & $\begin{array}{l}\text { Aconselha todas as vezes ou a maioria } \\
\text { das vezes os pacientes com padrões de } \\
\text { consumo de risco a diminuir ou parar } \\
\text { o consumo }\end{array}$ & $65,4 \%$ \\
\hline 4 & $\begin{array}{l}\text { Atendeu de }(0-5) \text { pacientes com } \\
\text { consumo pesado nos últimos } 12 \text { meses }\end{array}$ & $68,4 \%$ \\
\hline 5 & $\begin{array}{l}\text { Está pensando em fazer, mas ainda } \\
\text { não começou a realizar atividades } \\
\text { preventivas }\end{array}$ & $37,7 \%$ \\
\hline 6 & Não desenvolve atividades preventivas & $60,2 \%$ \\
\hline 7 & $\begin{array}{l}\text { Nunca realizou atividades ou } \\
\text { orientações preventivas }\end{array}$ & $62,2 \%$ \\
\hline
\end{tabular}

Foram feitas análises de associação entre cada questão do questionário de caracterização de práticas em saúde e os modelos de percepção obtidos a partir do questionário sobre modelo de percepção sobre problemas de saúde. Os modelos de percepção desse último questionário foram divididos em dois grupos, um formado pelos profissionais que apresentam o modelo moral de percepção e outro pelos profissionais que apresentam um dos três outros tipos de modelos de percepção, médico, compensatório ou iluminado. 
No que se refere à porcentagem estimada de pacientes atendidos com problemas relacionados ao uso de álcool, verificou-se uma mediana 20 para os profissionais que apresentam uma percepção moralizante do alcoolismo e uma mediana 10 para os profissionais que apresentam outro modelo de percepção. Não foi obtida diferença estatística pelo Teste Mann-Whitney.

Verificou-se que 56,6 \% dos profissionais que apresentam uma percepção moralizante do alcoolismo relata perguntar sobre o consumo de risco e a história dos problemas relacionados ao uso de álcool a maioria das vezes ou algumas vezes, enquanto $60,0 \%$ dos profissionais que apresentam outro modelo de percepção relata fazer tal pergunta a maioria das vezes ou algumas vezes.

No que se refere à frequência com que o profissional aconselha os pacientes com padrões de consumo de riscos para problemas relacionados ao uso de álcool a diminuir ou a parar com esse consumo, verificou-se que $62,5 \%$ dos profissionais que apresentam uma percepção moralizante do alcoolismo relata aconselhar todas as vezes ou a maioria das vezes, enquanto $65 \%$ dos profissionais que apresentam outro modelo de percepção relata também aconselhar todas as vezes ou a maioria das vezes.

Verificou-se que $70,1 \%$ dos profissionais que apresentam uma percepção moralizante do alcoolismo relata ter tratado dos problemas relacionados ao consumo pesado de álcool de (0-5) pacientes nos últimos 12 meses, enquanto $75,0 \%$ dos profissionais que apresentam outro modelo de percepção também relata ter tratado de (0-5) pacientes nesse período.
Tratando-se de atividades de prevenção aos problemas relacionados ao uso de álcool, verificou-se que $41,9 \%$ dos profissionais que apresentam uma percepção moralizante do alcoolismo se encaixa na proposição estou pensando em fazer, mas ainda não comecei, enquanto $47,4 \%$ dos profissionais que apresentam outro modelo de percepção marcaram a mesma alternativa.

Verificou-se que $70,3 \%$ dos profissionais que apresentam uma percepção moralizante do alcoolismo não desenvolve atividade(s) ou abordagens preventivas em relação ao consumo de álcool e outras drogas, enquanto $60,0 \%$ dos profissionais que apresentam outro modelo de percepção também não as desenvolvem.

Verificou-se ainda que $63 \%$ dos profissionais que apresentam uma percepção moralizante nunca realizou palestras ou orientações preventivas para o consumo de álcool ou outras drogas, enquanto $70 \%$ dos profissionais que apresentam outro modelo de percepção também nunca realizou tais atividades.

Não foi observada uma associação significativa no Teste Qui-quadrado ( $p>$ $0,05)$ para nenhuma das questões acima mencionadas. Dessa forma, não houve diferença estatisticamente significativa entre a prática de saúde relacionada ao consumo de álcool dos profissionais que apresentam o modelo de percepção moral do alcoolismo e a prática daqueles que apresentam outro modelo de percepção. Na Tabela 4, encontram-se os dados obtidos a partir das associações. 
Tabela 4. Associação entre práticas de saúde e modelo de percepção Brickman em profissionais de Atenção Primária $(n=170)$

\begin{tabular}{|c|c|c|c|}
\hline Questão & Resposta & $\begin{array}{l}\text { Modelo } \\
\text { Moral }\end{array}$ & $\begin{array}{l}\text { Modelo } \\
\text { não moral }\end{array}$ \\
\hline 1 & $\begin{array}{l}\text { Mediana da porcentagem } \\
\text { estimada de pacientes atendidos } \\
\text { com problemas relacionados ao } \\
\text { álcool }\end{array}$ & 20 & 20 \\
\hline 2 & $\begin{array}{l}\text { Pergunta sobre o consumo nas } \\
\text { consultas a maioria das vezes ou } \\
\text { algumas vezes }\end{array}$ & $56,6 \%$ & $60 \%$ \\
\hline 3 & $\begin{array}{l}\text { Aconselha todas as vezes ou a } \\
\text { maioria das vezes os pacientes } \\
\text { com padrões de consumo de risco } \\
\text { a diminuir ou a parar o consumo }\end{array}$ & $62,5 \%$ & $65 \%$ \\
\hline 4 & $\begin{array}{l}\text { Atendeu de }(0-5) \text { pacientes com } \\
\text { consumo pesado nos últimos } 12 \\
\text { meses }\end{array}$ & $70,1 \%$ & $75 \%$ \\
\hline 5 & $\begin{array}{l}\text { Está pensando em fazer, mas } \\
\text { ainda não começou atividades } \\
\text { preventivas }\end{array}$ & $41,9 \%$ & $47,7 \%$ \\
\hline 6 & $\begin{array}{l}\text { Não desenvolve atividades } \\
\text { preventivas }\end{array}$ & $70,3 \%$ & $60,0 \%$ \\
\hline 7 & $\begin{array}{l}\text { Nunca realizou atividades ou } \\
\text { orientações preventivas }\end{array}$ & $63 \%$ & $70,0 \%$ \\
\hline
\end{tabular}

\section{Discussão}

A hipótese de que a dependência de álcool seria uma condição de saúde estigmatizada pelos profissionais de saúde foi comprovada em nosso estudo, o que está em consonância com outros estudos que também apontam o alcoolismo como uma condição de saúde estigmatizada (Ronzani et al., 2009; Silveira et al., 2009; Peluso \& Blay, 2008; Palm, 2006; Corrigan et al., 2005).

A segunda hipótese, a de que a estigmatização se associa às práticas em saúde dos profissionais em relação ao consumo de álcool, não foi confirmada. Não houve diferença estatística entre as práticas de saúde dos profissionais que apresentam o modelo de percepção moral e aqueles que apresentam outro tipo de percepção do alcoolismo. Esses achados aparentemente contradizem a literatura, uma vez que esta aponta que a estigmatização de uma condição de saúde por parte dos profissionais de saúde interfere na qualidade de atendimento oferecido ao usuário (Ronzani \& Andrade. 2006: Berger, et al, 2005). 
Contudo, parece-nos que o questionário utilizado para avaliar as práticas dos profissionais em relação aos usuários de álcool, caracterização de práticas em saúde, se mostrou limitado para captar aspectos menos objetivos da prática, tais como qualidade do atendimento; portanto, a principal limitação do presente estudo foi a restrição do instrumento utilizado, que se refere predominantemente à frequência com que os profissionais realizam determinadas atividades referentes ao consumo de álcool. Outra limitação é que a amostra foi definida por conveniência, o que impossibilita a generalização dos dados e confere ao estudo um caráter sobretudo descritivo.

Apesar das referidas limitações, o estudo gerou contribuições ao constatar que os profissionais que moralizam a dependência de álcool realizam atividades em saúde relativas ao consumo de álcool com a mesma frequência que os profissionais que não moralizam essa dependência, embora a qualidade dessas atividades seja questionável (Ronzani \& Andrade, 2006; Berger, etal, 2005). Esse achado se mostra relevante para o planejamento, a implementação e a avaliação de políticas relacionadas ao consumo de álcool, uma vez que ressalta a importância de se entender o processo de estigmatização e suas implicações, aponta a necessidade de uma avaliação ampliada das práticas em saúde que não se restrinja à ocorrência das mesmas (Palm, 2006; Fortney, Mukherjee, Curran, Fortney, Han, \& Booth, 2004) e ressalta a importância de se promover qualificações profissionais centradas na mudança de atitudes quando relacionadas a problemas de saúde estigmatizados (Ronzani et al., 2009), considerando-se que o simples aprendizado e a incorporação na rotina de práticas em saúde voltadas para tais problemas não implica melhor qualidade de atendimento aos usuários e, em conseqüência, melhoria dos índices do problema de saúde em questão.

Outro achado que merece destaque refere-se às atividades preventivas em relação ao consumo de álcool. Apesar de a amostra ser constituída por profissionais da APS, setor que se destaca ou deveria destacar-se por suas ações voltadas para a prevenção/promoção à saúde (Ronzani \& Silva, 2008), mais de $60 \%$ dos profissionais, independentemente do modelo de percepção, relata não desenvolver atividades ou abordagens preventivas em relação ao consumo de álcool e outras drogas e nunca ter desenvolvido palestras ou orientações para o consumo de álcool ou outras drogas.

Tal achado aponta a necessidade mais ampla de uma formação dos profissionais para trabalhar dentro da perspectiva de prevenção e de promoção de saúde fora do problema de saúde em questão (Ceccim, 2005). Muitas vezes a APS constitui um modelo diferente e distante da formação dos profissionais que aí passam a atuar; outros profissionais, ainda, não contam com nenhum tipo de formação nem capacitação, como é o caso de parte dos agentes comunitários de saúde.

\section{Considerações finais}

Os profissionais da APS se mostram em uma posição estratégica para realizar práticas de prevenção ao consumo de risco de álcool, contudo, verifica-se a ausência de tais práticas bem como a estigmatização do uso de álcool por parte desses profissionais. Ressalta-se, portanto, a necessidade de capacitação desses profissionais para práticas de tratamento, reabilitação e, sobretudo, de prevenção relacionadas ao consumo de álcool que incluam não só o aprimoramento técnico como também a mudança de crenças e atitudes sobre usuários de álcool (Ronzani et al., 2009; Palm, 2006).

Devido à relevância do tema abordado, sugerese que estudos futuros avaliem as práticas dos profissionais de saúde de uma forma mais ampla, a fim de identificar como a estigmatização do problema de saúde em questão se associa a tais atividades. 
Agradecemos à Propesq/UFJF, Edital Consolidação de Grupos de Pesquisa, Fundação de Amparo à Pesquisa do Estado de Minas Gerais (FAPEMIG), Programa Pesquisador Mineiro e Edital Universal pelo apoio financeiro. Agradecemos ainda ao psicólogo Leonardo Fernandes Martins pela colaboração na análise de dados e aos acadêmicos de Psicologia Tamires Jordão Laport, Tassiana Cristina Mendes Miranda, Bianca Cristine Gomide Costa, Ana Cláudia Ferreira Cezario e às psicólogas Lívia Ferreira de Araujo, Érica Cruvinel e Daniela Cristina Mota Belchior pela colaboração na coleta de dados.

Marina Castro de Oliveira

Mestre em Saúde Coletiva pela Universidade Federal de Juiz de Fora. Pesquisadora do Centro de Referência em Pesquisa, Intervenção e Avaliação em Álcool e Drogas- CREPEIA- da Universidade Federal de Juiz de Fora- MG-Brasil. E-mail: marinacastro19@yahoo.com.br

\section{Telmo Mota Ronzani}

Pós-Doutorado pela Universidade de São Paulo. Bolsista de Produtividade CNPq. Professor adjunto da Universidade Federal de Juiz de Fora, Minas Gerais - MG - Brasil.

E-mail: telmo.ronzani@ufjf.edu.br

Endereço para envio de correspondência:

Rua Johann Strauss no 120, Bairro São Pedro, Juíz de Fora - Minas Gerais - MG - Brasil CEP: 38405-320

Recebido 29/4/2010, 1a Reformulação 24/10/2011, Aprovado 13/4/2012. 
Andrade, L. O. M., Barreto, I. C. H. C., \& Bezerra, R. C. (2006). Atenção Primária à Saúde e Estratégia Saúde da Família. In G. W. S. Campos, M. C. S. Minayo, M. Akerman, M. Drumond Junior, \& Y. M. de Carvalho (Orgs.), Tratado de Saúde Coletiva (pp. 783-836). São Paulo/Rio de Janeiro: Hucitec/ Fiocruz.

Babor,T., Higgins-Biddle, J. C., Saunders, J. B., \& Monteiro, M. G. (2001). AUDIT - the Alcohol Use Disorders Identification Test: Guidelines for use in primary care. Geneva: WHO.

Berger, M., Wagner, T. H., \& Baker, L. C. (2005). Internet use and stigmatized illness. Social Science \& Medicine, 61(8), 18211827.

Brasil. Ministério da Saúde. (2004). A política do Ministério da Saúde para a atenção integral a usuários de álcool e outras drogas. Brasília, DF: Ministério da Saúde.

Carlini, E. A., Galduróz, J. C. F., Noto, A. R., \& Nappo, S. A. (2005). II Levantamento domiciliar sobre uso de drogas no Brasil. Brasília, DF: Centro Brasileiro de Informações Sobre Drogas Psicotrópicas.

Ceccim, R. B.(2005). Educação permanente em saúde: desafio ambicioso e necessário. Interface - Comunicação, Saúde, Educação, 9(16),161-177.

Corrigan, P. W., Lurie, B. D., Goldman, H. H., Slopen, N., Medasani, K., \& Phelan, S. (2005). How adolescents perceive the stigma of mental illness and alcohol abuse. Psychiatric Services, 56(5), 544-550.

Escorel, S., Giovanella, L., Mendonça, M. H. M., \& Castro, M. M. S. (2007). The family health program and the construction of a new model for primary care in Brazil. Panamerican Journal of Public Health, 21(2-3), 164-176.

Fortney, J., Mukherjee, S., Curran, G., Fortney, S., Han, X., \& Booth, B. M. (2004). Factors associated with perceived stigma for alcohol use and treatment among at-risk drinkers. Journal of Behavioral Health \& Services Research, 31(4), 418-429.

Hoffmann, M. H., Carbonell, E., \& Montoro, L. (2006). Álcool e segurança - epidemiologia e efeitos. Psicologia: Ciência e Profissão, 16(1), 28-37.

Humeniuk, R., \& Poznyak, V. (2004). Intervenção Breve para o Abuso de Substâncias: Guia para Uso na Atenção Primária à Saúde. Genebra: OMS.

Instituto Brasileiro de Geografia e Estatística, IBGE. (2010). Cidades@ 2010. Recuperado em 01 dezembro, 2010 de <http://www.ibge.gov.br/cidadesat/topwindow.htm>.
Laranjeira, R., \& Meloni, J. N. (2004). Custo social e de saúde do consumo do álcool. Revista Brasileira de Psiquiatria, 26(1), 7-10.

Palm, J. (2006). Moral concerns - Treatment staff and user perspectives on alcohol and drug problems. Doctor's degree thesis, University of Stockholm, Stockholm.

Peluso, E. T. P., \& Blay, S. L. (2008). Public perception of alcohol dependence. Revista Brasileira de Psiquiatria, 30(1), 19-24.

Ronzani, T. M. (2005). Avaliação de um processo de implementação de estratégias de prevenção ao uso excessivo de álcool em serviços de atenção primária à saúde: entre o ideal e o possível. Tese de doutorado. Instituto de Psicobiologia, Universidade Federal de São Paulo, São Paulo, SP.

Ronzani, T. M., \& Andrade T. (2006). A estigmatização associada ao uso de substâncias como obstáculo à detecção, prevenção e tratamento. In Senad (Org.), Sistema para detecção de uso abusivo e dependência de substâncias psicoativas. (pp. 25-32). Brasília, DF: Senad.

Ronzani, T. M., Higgins-Biddle, J., \& Furtado E. F. (2009). Stigmatization of alcohol and other drug users by primary care providers in Southeast Brazil. Social Science \& Medicine, 69, 1080-1084.

Ronzani, T. M., \& Silva, C. M. (2008). O Programa Saúde da Família segundo profissionais de saúde, gestores e usuários. Ciência \& Saúde Coletiva, 13(1), 23-34.

Senad. (2007). I Levantamento Nacional sobre os Padrões de Consumo de Álcool na População Brasileira. Brasília, DF: Secretaria Nacional Antidrogas. Recuperado em 15 junho, 2009 de www.obid.senad.gov.br.

Silveira, P. S. (2010). Estigmatização do uso de álcool e outras drogas entre profissionais de saúde de Juiz de Fora. Dissertação de mestrado. Instituto de Psicologia, Universidade Federal de Juiz de Fora, Juiz de Fora, MG.

Silveira, P. S., Martins, L. F., \& Ronzani, T. M. (2009). Moralização sobre o uso de álcool entre agentes comunitários de saúde. Psicologia: Teoria e Prática, 11(1), 62-75.

World Health Organization. (1999). Global Status Report on Alcohol. Geneva: Autor

World Health Organization. (2007). Expert Committee on Problems Related to Alcohol Consumption. Geneva: Autor.

World Health Organization. (2003). The World Health Report: Shaping the future. Geneva: Autor 